\title{
hESC Adaptation, Selection and Stability
}

\author{
C. Grandela $\cdot$ E. Wolvetang
}

Published online: 22 May 2007

(C) Humana Press Inc. 2007

\section{Introduction}

Human Embryonic Stem Cells (hESC) are vigorously investigated as a source for cell replacement therapies. In order to deliver on their promise large amounts of high quality $\mathrm{hESC}$ will be needed that are genetically stable, free of animal products and manipulated to evade the immune system through either SCNT or other technologies. Here we will review several aspects of hESC biology that may directly or indirectly affect their genetic stability.

\section{hESC Culture Conditions}

Human embryonic stem (hESC) cell lines are established from the inner cell mass (ICM) of the blastocyst and differentiate into all cell lineages of the body [29, 31]. They were initially derived in 1998 and have enormous potential

\section{Grandela}

PDBEB - Programa Doutoral em Biologia Experimental

e Biomedicina, Department of Zoology,

Center for Neuroscience and Cell Biology,

University of Coimbra, Coimbra, Portugal

C. Grandela

Monash Institute of Medical Research, Monash University,

Clayton, Australia

\section{E. Wolvetang $(\bowtie)$}

Australian Stem Cell Centre, Level 3 North Building 75

(STRIP) Wellington Road, Clayton 3168 Victoria, Australia

e-mail: Ernst.Wolvetang@stemcellcentre.edu.au

\section{E. Wolvetang}

Department of Anatomy and Cell Biology, School of Biomedical

Sciences, Monash University, Clayton, Australia as a source of cells for cell replacement therapies and as a model for early human development [13]. Two culture conditions are widely used to propagate hESC. The standard method of culturing hESC stock cultures involves weekly mechanical passaging of morphologically undifferentiated appearing parts of a hESC colony onto full density $\left(6 \times 10^{4} \mathrm{cell} / \mathrm{cm}^{2}\right)$ mouse or human fibroblast feeder layers in a medium containing $20 \%$ FCS $[29,31]$. This protocol, albeit time, money and effort intensive, has proven to be a safe and reliable method for the long term propagation of hESC. This is highlighted by the fact that hESC thus cultured have never been reported to develop genetic abnormalities during culture. However, the major drawback of this method is the low number of hESC available for experimentation. Most laboratories therefore opt to maintain hESC stock cultures using this standard method and regularly establish fresh bulk cultures from these stocks for experimentation [2]. Bulk culture of hESC is performed according to a method developed by Amit et al. which involves passaging of enzymatically (collagenase, trypsin, etc) or non-enzymatically (cell dissociation solution, EDTA, etc) dissociated clumps of hESC on $1 / 3$ density feeders $\left(2 \times 10^{4} \mathrm{cell} / \mathrm{cm}^{2}\right)$ in KSOR with $20 \%$ serum replacement. This method indeed allows for dramatic expansion of hESC (Fig. 1). However, a number of reports have indicated that this is accompanied by an increase in genetic instability, methylation changes and mitochondrial mutations, abnormalities also seen in cancer and Embryo Carcinoma (EC) cells (the transformed counterpart of hESC) $[5,8,24]$. Since large amounts of undifferentiated stem cells of high quality will be needed for regenerative medicine approaches in the future, a deeper understanding of the molecular mechanisms that control the genetic stability of hESC is required. In addition culture methods that prevent the occurrence of genetic instability and/or 
Fig. 1 Comparison of "Standard" culture method with "Bulk"-culture method. See text for detailed explanation

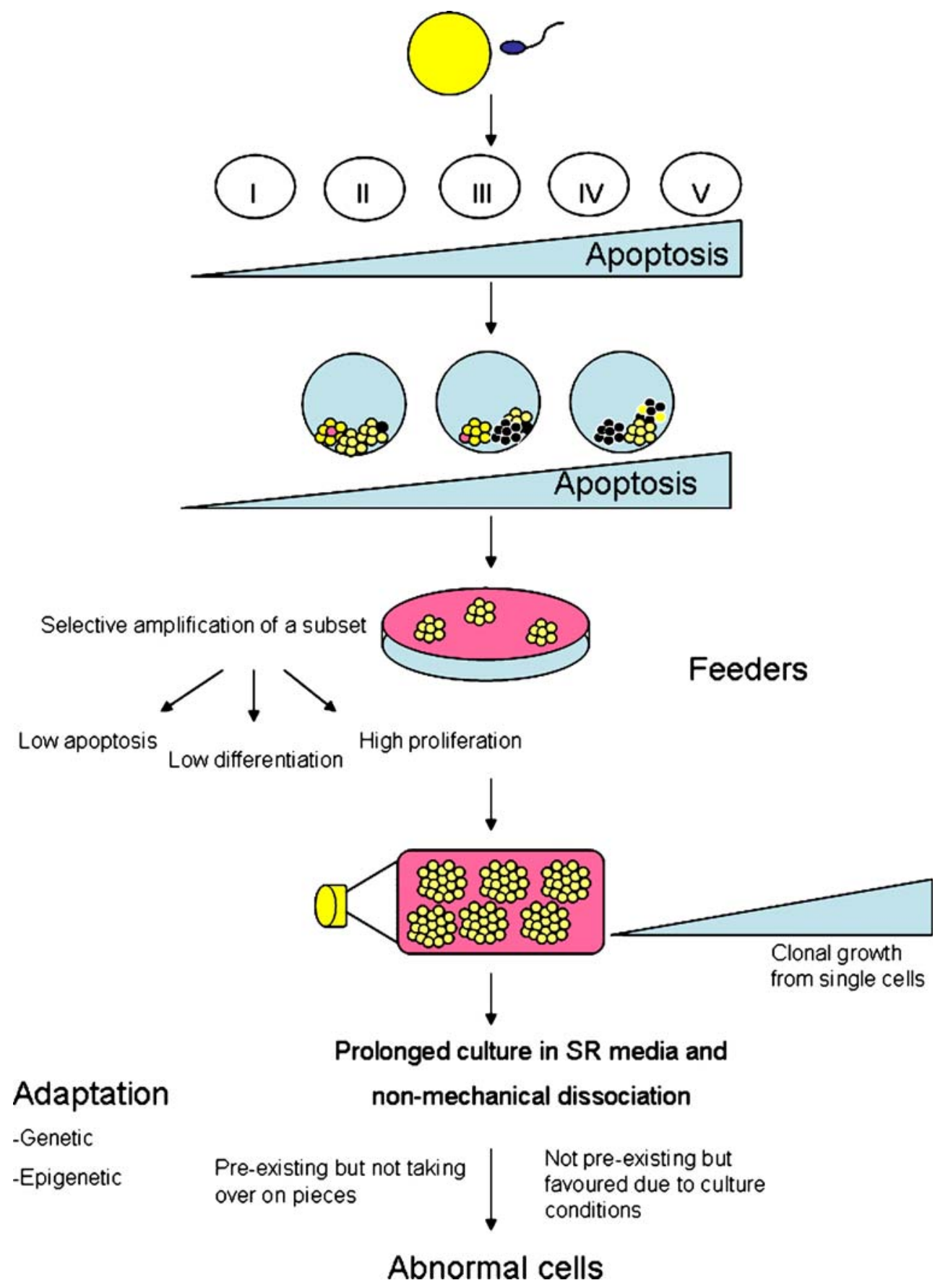

technologies to purge abnormal hESC from large scale hESC cultures will have to be developed.

\section{Pre-existing versus Acquired Genetic Abnormalities?}

The fact that hESC cultured under standard conditions display such remarkable genetic stability (normal karyotypes for over 200 weekly passages) as compared to bulk cultured cells (frequent abnormal karyotypes between passage 20 and 60) suggests fundamental differences between these two culture systems. One can envisage two broad possible explanations for the occurrence of genetic abnormalities in bulk cultured hESC. (1) Bulk culture directly or indirectly causes genetic instability (2) Genetic instability is an intrinsic feature of $\mathrm{hESC}$ in general but the phenotype of genetically abnormal cells only becomes apparent in bulk cultured hESC. If (1) is correct and the abnormalities occur as a consequence of the bulk culture conditions then bulk cultured hESC should be found to 
exhibit a combination of increased mutagenesis, reduced apoptosis, reduced DNA-repair and enhanced growth/ survival properties as compared to hESC grown in standard conditions. If (2) is correct and $\mathrm{hESC}$ grown under standard conditions show similar intrinsic genetic instability as bulk cultured hESC then one would predict that standard grown hESC exhibit more efficient mechanisms to delete/suppress abnormal cells, display enhanced repair DNA or that the acquired genetic abnormalities do not confer sufficient growth/survival enhancement under standard conditions to take over the culture. In order to test these hypotheses described above CGH arrays, SNP arrays and high through put whole genome sequencing will have to be employed in the future because the method that is most widely used to assess genetic stability of hESC at present is G-banding karyotype analysis. This method is only a low resolution assay, as $5-10 \%$ of all cells analysed need to show an abnormal karyotype to call the hESC genetically abnormal and does not identify small deletions or mutations.

\section{Can We Phenotypically Distinguish Genetically Normal and Abnormal hESC?}

Embryocarcinoma (EC) cells are the transformed genetically unstable counterpart of hESC and characteristically display very similar cytogenetic abnormalities to hESC with acquired genetic abnormalities such as trisomy 12,17 and $X[5,8,24]$. How then does one define a genetically healthy hESC? Some would argue that it is the ability to form teratomas with representatives of all three germ lineages that identifies the stem cell. However, EC cells also exhibit this property with the difference that undifferentiated primitive cells are still present in the graft that can be transplanted to new recipients, identifying them as a teratocarcinoma. However, since this particular experiment is usually not performed with hESC teratomas it is difficult to determine whether the hESC were actually stem cells or EC cells. Indeed, in the majority of reports there are only high power images of differentiated hESC shown and no overview of what other (undifferentiated?) cell types are present in such grafts. Others would argue that the expression of stem cell markers is proof of the presence of hESC. However, EC cells also express pluripotency markers and hESC with genetic abnormalities, such as ones that are marked by CD30 (see section below), actually display higher marker expression $(>95 \%)$ than genetically healthy hESC (>70-80\%) which usually display $15-20 \%$ spontaneous background differentiation. The presence of a fraction of spontaneously differentiating hESC in culture may therefore be a better indication of a genetically healthy hESC culture than hESC that express unusually high levels of pluripotency markers. This phenomenon is exacerbated by the high proliferative potential of high pluripotency marker expressing hESC. Indeed our data indicate that the more undifferentiated a hESC line is, the faster it grows (Filipczyk et al. pers. comm.) Unfortunately this also applies to hESC with abnormally high pluripotency marker expression that more closely resemble EC cells. Such cells would therefore rapidly take over the culture and without good assays to discriminate hESC from EC cells or from ES cells with genetic alterations that do not show up in Gbanding karyotype analyses one could easily interpret the properties of such genetically compromised cells for those of hESC cells.

\section{Selective Pressures During Establishment of HES Cell Lines}

Since we can not rule out the possibility that genetically compromised hESC are present from the inception of a hESC culture and because genetically abnormal hESC have a growth/survival/differentiation advantage over genetically "healthy" hESC it may be informative to consider the history of the cells and the selective pressures placed upon $\mathrm{hESC}$ cultures during their establishment and subsequent propagation (Fig. 2). hESC are cells isolated from the inner cell mass of day 5 blastocysts procured from to be discarded excess IVF embryos [29, 31]. The first decision in the history of a hESC line is therefore made at the oocyte stage when good quality oocytes are selected for IVF. In general the best fertilized cells are selected for implantation while the left over eggs with a potentially lower quality score can then made available for stem cell research. Post fertilization only some of the excess zygotes subsequently progress to blastocyst stage for reasons that are not fully understood at present. The next step in the establishment of a hESC line involves the selection of blastocysts with a high standard scoring classification (i.e. symmetry and low levels of cell fragmentation (apoptosis). Apoptosis of ICM cells is thought to be part of a quality control program perhaps aimed at eliminating abnormal cells from the developing embryo. This is supported by the observation that the incidence of apoptosis in in vitro produced embryos is increased by suboptimal culture conditions. Indeed, 7$8 \%$ of cells in pre-implantation embryos undergo apoptosis during this time and this occurs mainly in the ICM [11]. Nevertheless, early human development is accompanied by a high degree of genetic abnormality, with one third of first trimester miscarriages attributed to chromosomal defects [23]. To our knowledge to date there has been no systematic investigation into the potential correlation between the level of ICM apoptosis at the blastocyst stage and their subsequent ability to establish hESC lines or how this relates to their long term genetic stability. However, 
Fig. 2 The sequence of potential selective pressures during the establishment of a hESC culture. Excess oocytes not selected for reproduction are made available for hESC establishment. A subset of fertilized embryos progresses to blastocyst stage. Inner cell mass cells are selected for low apoptotic index. A subset of hESC proceeds to form a cell line after immunosurgery. A subset of hESC responds to MEF secreted maintenance factors and media components. Further selection of hESC occurs during culture

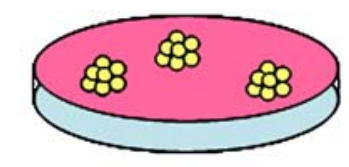

- $6 \times 10^{4}$ cells $/ \mathrm{cm}^{2}$ Feeders

-Serum containing medium

-Mechanical passaging
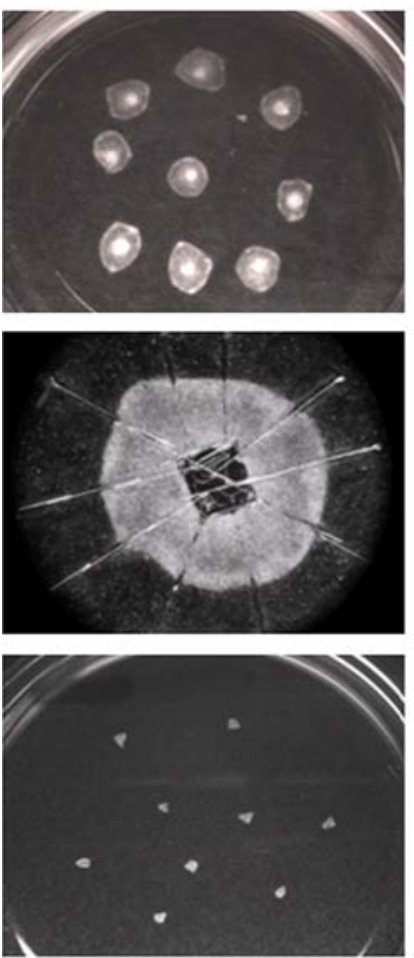

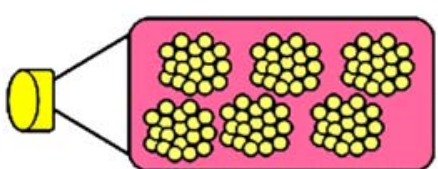

- $2 \times 10^{4}$ cells $/ \mathrm{cm}^{2}$ Feeders

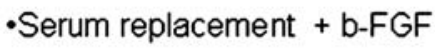

-Non-mechanical passaging (enzymatic or non-enzymatic)

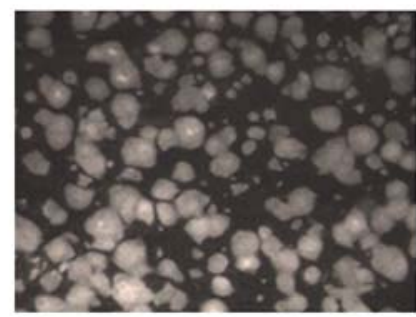

Overview of plate (day 7)

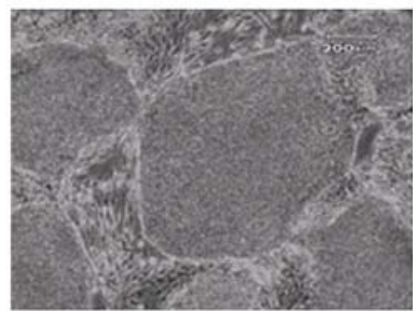

Colony detail

(at passaging)

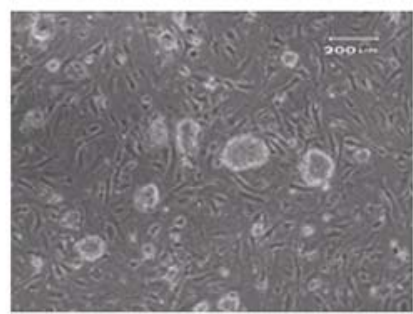

Overview of plate (day 1 after passaging) several studies have correlated the occurrence of chromosome abnormalities in SCNT embryos with the frequency of abnormalities in the donor cell line [22]. Donor cell lines with a higher percentage of genetically abnormal cells were found to cause a higher rate of chromosomally abnormal blastomeres in the SCNT embryos. It would be interesting to determine whether donor nuclei with chromosomal amplifications also detected in hESC (such as trisomy 12, 17 , or $\mathrm{X}$ ) show an enhanced ability to establish ES-cell lines. Following immuno-surgery and isolation of the inner cell mass, the next stage of hES derivation involves the propagation of isolated clumps of inner cell mass cells after plating on full density mouse or human fibroblast feeder layers. Again only a subset of these clumps can be coaxed into becoming a hESC line with rather low frequency. It seems self-evident that during this step there is a selection for cells that can respond to the maintenance factors excreted by the feeder layers and the medium components that aid in the establishment of hESC lines. In addition one would select for hESC that exhibit low apoptosis and a low propensity to differentiate since these are the cells that have the highest proliferative capacity. There is a large body of circumstantial evidence to suggest that mES lines adapt to their culture conditions and that various strains or substrains differ in their ability to generate ES cell lines or to contribute to the germline. Indeed, some mouse strains appear unable to establish mES lines, although the exact rationale for this phenomenon remains unknown. Although these parameters are not easy to assess in a human context it is not unreasonable to infer that a similar process occurs with hESCs. In addition there is a school of thought that argues that this selection is related to the occurrence of specific genetic and epigenetic changes that may aid in this adaptation to in vitro culture. As argued above hESC may have an innate ability to adapt to culture conditions or that certain culture conditions select for hESC with properties that allow propagation in a particular culture method. Since hESCs generally do not proliferate as single cells but rather as small clumps there is a possibility that hESC lines are intrinsically heterogeneous for this very reason from their 
inception. Indeed, the vast majority of hESC lines have not been derived from a single clone. An in depth analysis of the genetic heterogeneity within hESC lines is further complicated by the fact that hESCs cultured under the standard most optimal culture conditions rapidly become a heterogeneous population of cells when analysed at the gene expression level. Transcriptome analysis of gene expression in hESC stratified on the basis of their expression of known pluripotency marker expression shows that hESCs are not only in a constant flux of spontaneous differentiation, as judged by the presence of $20 \%$ of differentiated cells under these conditions, but indeed display substantial differences in mRNA expression [19] as compared to hESC with lower, but still positive, expression of the pluripotency markers. Indeed, transcriptome analysis of FACS sorted hESC stratified on the basis of the highest expression of pluripotency markers indicates that that these ultra-stem cells already express supposedly lineage specific markers such as nestin and brachyury by array analysis and RT-PCR, albeit at low levels.

\section{Chromosomal Abnormalities and Potential Significance}

Although the original reports on HESCs indicated that they can maintain a normal diploid karyotype, there is evidence that this depend on culture conditions. Different laboratories have reported that HESCs cultured for long periods of time tend to gain chromosomal abnormalities, resembling the ones observed in hEC cells. The most frequently observed karyotypic abnormalities are gain of chromosome $17 q, 12$ and $X$ chromosome [5, 8, 24], while there also seems to be a high proportion of partial or total chromosome 1 duplication. It has been postulated that increased dosage of those chromosomes might give the cells some sort of selective advantage.

Draper et al. [8] were the first to report gain of chromosome 17q and chromosome 12 in $\mathrm{H} 7$ and $\mathrm{H} 14$ cell lines cultured with Knockout SR replacer (Invitrogen/ GIBCO) instead of foetal calf serum and passaged with an enzymatic method (collagenase IV). They performed interphase fluorescence in situ hybridization (FISH) and showed that after 22 passages trisomy 17 was observed in $76 \%$ of the cells and after an additional 17 passages, in $95 \%$ of the cells, again indicating that such cells possess enhanced survival or proliferation properties. Buzzard and colleagues [6] cultured six NIH-registered HESC lines (hES1-6) between 34 and 140 passages using mechanical method of passaging cells rather than enzymatic or chemical methods of cell dissociation. They reported just one karyotype abnormality was detected in an early passage hES5, demonstrating that HESCs are not necessarily predisposed to karyotypic abnormalities even grown for extended time. Another group studied 3 cell lines (BG01, BG02 and BG03) and showed that karyotypic changes also give rise to differences in expression of genes, especially those involved in pluripotency [24]. They reported that bulk passaging (nonenzymatic- Cell Dissociation Buffer- or enzymatic- colagenase, trypsin) methods can compromise the genetic stability of HESCs, in contrast with manual methods. The bulk passage methods can be used for shorter periods (<passage 15) without problems.

Because classical G-band karyotype can only give a rough idea of chromosomal stability, techniques with more resolution should be used to detect smaller chromosomal and gene alterations/mutations. So other studies attempt to overcome that problem by using techniques, like SNP, to track down these alterations on HESCs, especially those that are cultured for long periods of time. Maitra and colleagues [21] compared 9 hESCs lines (BG01, BG02, BG03, H1, H7, H9, HES-2, HES-3, SA001 and SA002) derived and cultured in different laboratories at both early and late passage and found the former ones can develop one or more genomic alterations commonly observed in human cancers, mitochondrial DNA sequence mutations and gene promoter methylation. Enver et al. [9] investigated the adaptation process by comparing early passage (normal) and late passage (adapted) sublines of H7. The karyotype of early passage cells was normal, whereas the adapted showed an extra copy of chromosome 1 and chromosome 17q amplification. Slightly higher proportion of SSEA3+ cells were found in the adapted cultures as compared to the normal ones ( 83 and $64 \%$, respectively). They also showed failure in X-inactivation, suggesting that epigenetic changes are also associated with adaptation. Thus the mechanism of adaptation may be similar to the early events involved in oncogenesis and tumor progression. Baker et al. [3] have made an attempt to link culture adaptation and malignancy. In agreement with other laboratories they also observed a nonrandom gain of certain regions in the three mainly affected chromosomes in HESC $(12,17$ and $\mathrm{X})$. They hypothesized that these chromosomes may contain candidate genes that could be important for the observed culture adaptation and/or play a role in stem cell maintenance in vitro and cell tumorogenesis in vivo. One of these candidate genes present in terminal half of $\mathrm{q}$ arm of chromosome 17 is BIRC5, also known as Survivin. Survivin can act as an antiapoptotic gene and also plays a role in chromosome segregation. Survivin is expressed in a large number of tumor types such as neuroblastomas and associated with highest-risk tumors and poor survival outcome. Gain of chromosome 12 was also frequently observed in both HESCs and testicular germ cell tumors (TGCTs). Nanog, a pluripotency gene, is located on 12p13 and overexpression of this gene promotes self-renewal and prevents differentiation and thus can give hESC an 
anti-differentiation and proliferation advantage in culture. Another amplified region on chromosome 12, 12p11.2-12 that is also frequently amplifies in TGCTs harbours the oncogene K-RAS and SOX5, a gene involved in cell fate determination. Reduced apoptosis has been observed in malignant cells with this amplification and it is possible that amplification of this region may confer a similar survival advantage to hESC. Multiple studies have found that hESC acquire additional copies of chromosome $\mathrm{X}$ and often TGCTs have more than one $\mathrm{X}$ chromosome. Enver et al. further identified ELK1, A-RAF, the androgen receptor and its interacting protein NONO, as potential candidate genes on $\mathrm{X}$ that could be involved in hESC adaptation.

The non random gain of chromosomes 12, 17 and $\mathrm{X}$ observed in different hESC lines in different laboratories around the world lends support to the hypothesis that there may be genes on these chromosomes that confer a growth or anti-differentiation advantage to hESC in culture. Generating small clumps or single cells from hESC colonies in general leads to apoptosis and differentiation. The fact these abnormalities only occur in bulk cultured cells suggests that the seeding of small clumps of $\mathrm{hESC}$ puts selective pressure on hESC that favours hESC with such genetic abnormalities. In this context increased expression of genes that inhibit apoptosis (and differentiation) of hESC, such as CD30, prior to overt genetic abnormalities may increase the likelihood of acquiring further abnormalities.

\section{hESC and CD30}

We discovered that hESC cultured under bulk culture conditions rapidly start to express the CD30 antigen, a molecule that marks embryo carcinoma and Hodgkin lymphoma cells [7]. Overexpression of CD30 in Hodgkin lymphoma leads to ligand independent activation of downstream signalling activating MEK-ERK, AKT and NF-k-B pathways. Interestingly, these pathway were also identified as important regulators of stem cell maintenance. Moreover, CD30 may be directly involved in the genetic stability and transformation of hESC since it's overexpression leads to transformation of rat fibroblasts [26]. In agreement with a possible role of CD30 in hESC genetic stability, we previously were able to show that CD30 is a biomarker for genetically abnormal hESC and causes both increased NF-k-B activity and inhibition of apoptosis and is associated with b-FGF independent expression of pluripotency markers. It remains to be established whether CD30 expression is a bystander/indicator of these phenotypes or is responsible for all or some of these properties. Importantly, preliminary data from our laboratory indicate that $\mathrm{CD} 30$ expressing hESC display a reduced expression of p53 and an increase in survivin (BRC5) expression, suggesting that signalling downstream of CD30 may affect the expression of genes involved in genetic stability and chromosome segregation. An example of how CD30 expressing hESC phenotypically differ from CD30 negative hESC is uncovered when hESC are cultured under feeder free conditions. Feeder free HESC cultures involves seeding of bulk-culture grown hESC on matrigel coated dishes in the presence of feeder conditioned medium [34]. This has the advantage that it removes the physical interaction with the feeder layers and can thus be used to identify the growth factors excreted by the feeders that evidently are able to maintain hESC undifferentiated growth. It is however important that the hESC used in such methods are early passage hESC that have not undergone changes such as b-FGF independence and CD30 expression since in our experience genetically abnormal hESC or hESC that highly express CD30 have lost the ability to spontaneously differentiate in the absence of the feeder conditioned medium over a 2 week period and can thus no longer be used to identify maintenance factors. This highlights the importance of testing hESC cultured on matrigel with feeder conditioned medium for spontaneous differentiation in the absence of conditioned medium before claiming the identification of factors that maintain hESC in the absence of feeders.

\section{Epigenetics and Genomic Stability}

As was shown by Jaenisch et al., using an elegant model of transient DNMT1 inactivation in mouse ES-cells to generate imprint free ES cells, loss of methylation in ES cells is directly related to tumorigenesis [15]. Loss of imprinting leads to a higher growth rate, a shortened cell cycle time, cellular immortality, resistance to TGF $\beta$, and foci formation on a confluent monolayer. Consistent with this, imprint free-MEFs formed tumors in SCID mice and adult chimeric mice derived from IF-ES cells developed tumors in multiple tissues that were originally derived from the IF-ES cells. It appears therefore that imprinting plays a much wider role in tissue homeostasis than just regulating normal embryonic growth and is directly responsible for providing critical tumor suppressor activity in the mature organism. The next challenge will be to identify the genes whose methylation status/silencing is critical for this function. Igf $2 / \mathrm{H} 19$ was previously indicated but was variably methylated in the Jaenisch study. It appears however that loss of imprinting confers immortality to MEFs by inactivating the Arf-p53 pathway, but the mechanisms responsible remain to be elucidated. In light of these recent discoveries it is interesting to note that human ES cells were found to undergo a range of epigenetic modification upon prolonged culture in serum 
free culture conditions, including dramatic changes in methylation.

\section{miRNA in hESCs}

microRNAs (miRNAs) are an abundant class of endogenous non-protein-coding small RNAs, which negatively regulate gene expression at the posttranscriptional level in many developmental and metabolic processes. miRNAs regulate a variety of biological processes, including developmental timing, signal transduction, tissue differentiation and maintenance, disease, and carcinogenesis. It is increasingly recognized that miRNAs also play an essential role in stem cell self-renewal and differentiation. Some miRNAs are specifically expressed in stem cells and could be involved in controlling stem cell selfrenewal and differentiation by negatively regulating the expression of certain key genes in stem cells. Dicer, an enzyme complex involved in miRNA processing, is essential for mammalian development, with Dicer-deficient mice dying at embryonic day 7.5 with a lack of detectable multipotent stem cells $[18,24]$. Dicer loss compromises maturation of miRNAs and leads to a defect in gene silencing triggered by long dsRNAs. However, the absence of Dicer does not affect the ability of small interfering RNAs to repress gene expression. Importantly, Dicer loss compromises the proliferation of ES cells, which may explain the phenotype of DICER null mice. DGCR8 is another enzyme involved in nuclear processing of pre-miRNA. Analysis of DGCR8 mouse knockout ES cells shows that DGCR8 is essential for biogenesis of miRNAs [33]. On the induction of differentiation, DGCR8-deficient ES cells do not fully downregulate pluripotency markers and retain the ability to produce ES cell colonies. Thus miRNAs appear to play a role in the silencing of ES cell self-renewal that normally occurs with the induction of differentiation. There is evidence to suggest that shRNA are able to be transported/diffuse through GAP junctions between hESC. This opens up the interesting possibility that hESC may also be signalling to each other at a gene regulation level leading to a communal effect between multiple stem cells connected by GAP junctions. It remains to be established whether this phenomenon could explain the inability of hESC to grow from single cells and how this relates to the role of miRNAs in differentiation and self renewal.

\section{The Role of p53 in hESC}

DNA damage response and cell-cycle regulation differ markedly between mouse embryonic stem cells (mESCs) and somatic cells [16]. ES cells require sensitive mechanisms to maintain genomic integrity and do so, in part, by suppressing spontaneous mutation. Spontaneous mutation frequency in somatic cells is approximately $10(-4)$ compared with $10(-6)$ for mESCs cells. mESCs cells also lack a G1 checkpoint and are hypersensitive to IR and other DNA-damaging agents [16]. These characteristics facilitate apoptosis and the removal of cells with a mutational burden from the population, thereby keeping the population free of damaged cells. Nucleotide excision repair in mESCs exposed to genotoxic effects of UV-induced DNA damage is not very efficient and leads to induction of apoptosis [32], further indicating that ES cells favor the induction of apoptosis to avoid accumulation of transformed cells.

Array studies indicate that p53 and other genes involved in apoptosis are expressed in on hESC and $\operatorname{mESC}[3,10$, 27]. Array analysis of $\mathrm{H} 1$ cells showed [10] that ES cells express p53, MDM2 a negative regulator of $\mathrm{p} 53$, the proapoptotic downstream target of p53 Bax, as well as the $\mathrm{BAX}$ related genes $\mathrm{BAK}, \mathrm{HRK}$ and the $\mathrm{Bcl}-2$ family member MCL-1. Therefore the basic machinery for cytochrome c release and apoptosome activation is present in hESC.

P53 is considered a critical regulator of genomic integrity because of its ability to trigger apoptosis and/or cell cycle arrest in response to genotoxic stress. Indeed, mutations of the $p 53$ gene occur in approximately $50 \%$ of human cancers $[14,17,20]$. P53 can be involved in maintaining genetic stability in several ways. P53 exerts control over both the $\mathrm{G} 2 / \mathrm{M}$ and the $\mathrm{G} 1$ cell cycle checkpoints. P53 inactivation can synergize with oncogene activation leading to aneuploidy and chromosome imbalance. We speculate that if p53 pathway is altered in stem cells and an oncogene gets activated, these cells may have a survival or growth advantage and take over the culture. p53 can also affect genetic stability through it's ability to regulate centrosome duplication via either transactivation dependent mechanisms involving $\mathrm{p} 21$, or transactivation independent mechanisms, by interacting directly with other centrosomal proteins at the centrosome. Indeed, Survivin, an inhibitor of apoptosis and a chromosomal passenger protein whose deregulation leads to aneuplody, is negatively regulated by $\mathrm{p} 53$. As mentioned earlier, Survivin is also one of the candidate genes on the frequently duplicated chromosome 17 and a gene upregulated in CD30 expressing hESC (Wolvetang pers.comm.).

The precise role of p53 in both mouse and human ES cell apoptosis remains unclear. While some argue that p53 is non-functional in undifferentiated mESCs [1] others show that p53 does have a role to play in both UV-induced apoptosis [7, 35] as well as differentiation [29]. Qin et al. [20] have recently shown that HESCs undergo p53 mediated-apoptosis induced by UV [27]. They further 
reported that relatively high rate of spontaneous apoptosis of hESC in culture (10-15\% daily) is controlled by p53. At present it remains to be established whether this spontaneous apoptosis is due to suboptimal culture conditions or is an intrinsic feature of hESC that is involved in eliminating cells with acquired DNA damage.

\section{Concluding Remarks}

The development of genomic instability is central feature of carcinogenesis and is difficult to study in a cell lines because the molecular pathways involved in transformation are altered during the establishment of a cell line. The striking difference in genomic stability between hESC cultured under standard conditions and hESC expanded in "bulk" cultures offers a unique opportunity to study this process in a non-transformed model system. We believe that a better understanding of the interrelationship between selection/adaptation and genetic/epigenetic regulation in hESC will further our insight into the molecular processes that control genomic stability and transformation. The standardisation of assays to assess these parameters and the standardisation of culture methods for hESC through international collaborative initiatives will be critical for the advancement of this body of knowledge.

\section{References}

1. Aladjem, M. I., Spike, B. T., Rodewald, L. W., Hope, T. J., Klemm, M., Jaenisch, R., et al. (1998). ES cells do not activate p53dependent stress responses and undergo p53-independent apoptosis in response to DNA damage. Current Biology, 8, 145-155.

2. Amit, M., Carpenter, M. K., Inokuma, M. S., Chiu, C. P., Harris, C. P., Waknitz, M. A., et al. (2000). Clonally derived human embryonic stem cell lines maintain pluripotency and proliferative potential for prolonged periods of culture. Developments in Biologicals, 227, 271-278.

3. Baker, D. E., Harrison, N. J., Maltby, E., Smith, K., Moore, H. D., Shaw, P. J., et al. (2007) Adaptation to culture of human embryonic stem cells and oncogenesis in vivo. Nature Biotechnology, 25, 207-215.

4. Bhattacharya, B., Cai, J., Luo, Y., Miura, T., Mejido, J., Brimble, S. N., et al. (2005). Comparison of the gene expression profile of undifferentiated human embryonic stem cell lines and differentiating embryoid bodies. BMC Developmental Biology, 5, 22.

5. Brimble S. N., Zeng, X., Weiler, D. A., Luo, Y., Liu, Y., Lyons, I. G., et al. (2004). Karyotypic stability, genotyping, differentiation, feeder-free maintenance, and gene expression sampling in three human embryonic stem cell lines derived prior to August 92001. Stem Cells Development 13, 585-597.

6. Buzzard, J. J., Gough, N. M., Crook, J. M., Colman, A. (2004) Karyotype of human ES cells during extended culture. Nature Biotechnology, 22, 381-382

7. Chao, C., Saito, S., Kang, J., Anderson, C. W., Appella, E., \& Xu, Y. (2000). p53 transcriptional activity is essential for p53-dependent apoptosis following DNA damage. EMBO Journal, 19, 4967-4975.
8. Draper, J. S., Smith, K., Gokhale, P., Moore, H. D., Maltby, E., Johnson, J., et al. (2004) Recurrent gain of chromosomes 17q and 12 in cultured human embryonic stem cells. Nature Biotechnology, 22, 53-54.

9. Enver, T., Soneji, S., Joshi, C., Brown, J., Iborra, F., Orntoft, T., et al. (2005) Cellular differentiation hierarchies in normal and culture-adapted human embryonic stem cells. Human Molecular Genetics, 14, 3129-3140.

10. Ginis, I., Luo, Y., Miura, T., Thies, S., Brandenberger, R., GerechtNir, S., et al. (2004). Differences between human and mouse embryonic stem cells. Developments in Biologicals, 269, 360-380.

11. Hardy, K., Handyside, A. H., \& Winston, R. M. (1989). The human blastocyst: Cell number, death and allocation during late preimplantation development in vitro. Development, 107, 597-604.

12. Herszfeld, D., Wolvetang, E., Langton-Bunker, E., Chung, T. L., Filipczyk, A. A., Houssami, S., et al. (2006). CD30 is a survival factor and a biomarker for transformed human pluripotent stem cells. Nature Biotechnology, 24, 351-357.

13. Hoffman, L. M., \& Carpenter, M. K. (2005). Characterization and culture of human embryonic stem cells. Nature Biotechnology, 23, 699-708.

14. Hollstein, M., Sidransky, D., Vogelstein, B., \& Harris, C. C. (1991). p53 mutations in human cancers. Science, 253, 49-53.

15. Holm, T. M., Jackson-Grusby, L., Brambrink, T., Yamada, Y., Rideout, W. M., 3rd, \& Jaenisch, R. (2005). Global loss of imprinting leads to widespread tumorigenesis in adult mice. Cancer Cell, 8, 275.

16. Hong, Y., \& Stambrook, P. J. (2004). Restoration of an absent G1 arrest and protection from apoptosis in embryonic stem cells after ionizing radiation. Proceedings of the National Academy of Sciences of the United States of America, 101, 14443-14448.

17. Hussain, S. P., \& Harris, C. C. (1998). Molecular epidemiology of human cancer: contribution of mutation spectra studies of tumor suppressor genes. Cancer Research, 58, 4023-4037.

18. Kanellopoulou, C., Muljo, S. A., Kung, A. L., Ganesan, S., Drapkin, R., Jenuwein, T., et al. (2005). Dicer-deficient mouse embryonic stem cells are defective in differentiation and centromeric silencing. Genes \& Development, 19, 489-501.

19. Laslett, A. L., Grimmond, S., Gardiner, B., Stamp, L., Lin, A., Hawes, S. M., et al. (2007). Transcriptional analysis of early lineage commitment in human embryonic stem cells. $B M C$ Developmental Biology, 7(1), 12.

20. Levine, A. J. (1997). p53, the cellular gatekeeper for growth and division. Cell, 88, 323-331.

21. Maitra, A., Arking, D. E., Shivapurkar, N., Ikeda, M., Stastny, V., Kassauei, K., et al. (2005) Genomic alterations in cultured human embryonic stem cells. Nature Genetics, 37, 1099-1103.

22. Mastromonaco, G. F., Perrault, S. D., Betts, D. H., \& King, W. A. (2006). Role of chromosome stability and telomere length in the production of viable cell lines for somatic cell nuclear transfer. BMC Developmental Biology, 6, 41.

23. Metcalfe, A. D., Hunter, H. R., Bloor, D. J., Lieberman, B. A., Picton, H. M., Leese, H. J., et al. (2004). Expression of 11 members of the BCL-2 family of apoptosis regulatory molecules during human preimplantation embryo development and fragmentation. Molecular Reproduction \& Development, 68, 35-50.

24. Mitalipova, M. M., Rao, R. R., Hoyer, D. M., Johnson, J. A., Meisner, L. F., Jones, K. L., et al. (2005) Preserving the genetic integrity of human embryonic stem cells. Nature Biotechnology, 23, 19-20.

25. Murchison, E. P., Partridge, J. F., Tam, O. H., Cheloufi, S., \& Hannon, G. J. (2005). Characterization of Dicer-deficient murine embryonic stem cells. Proceedings of the National Academy of Sciences of the United States of America, 102, 12135-12140. 
26. Nonaka, M., Horie, R., Itoh, K., Watanabe, T., Yamamoto, N., \& Yamaoka, S. (2005). Aberrant NF-kappaB2/p52 expression in Hodgkin/Reed-Sternberg cells and CD30-transformed rat fibroblasts. Oncogene, 24(24), 3976-3986.

27. Qin, H., Yu, T., Qing, T., Liu, Y., Zhao, Y., Cai, J., et al. (2006). Regulation of apoptosis and differentiation by p53 in human embryonic stem cells. Journal of Biological Chemistry, 282, 5842-5852.

28. Ramalho-Santos, M., Yoon, S., Matsuzaki, Y., Mulligan, R. C., \& Melton, D. A. (2002). "Stemness": transcriptional profiling of embryonic and adult stem cells. Science, 298, 597-600.

29. Reubinoff, B. E., Pera, M. F., Fong, C. Y., Trounson, A., \& Bongso, A. (2000). Embryonic stem cell lines from human blastocysts: somatic differentiation in vitro [see comment][erratum appears in Nat Biotechnol 2000 May;18(5):559]. Nature Biotechnology, 18, 399-404.

30. Sabapathy, K., Klemm, M., Jaenisch, R., \& Wagner, E. F. (1997). Regulation of ES cell differentiation by functional and conformational modulation of p53. EMBO Journal, 16, 6217-6229.
31. Thomson, J. A., Itskovitz-Eldor, J., Shapiro, S. S., Waknitz, M. A., Swiergiel, J. J., Marshall, V. S., et al. (1998). Embryonic stem cell lines derived from human blastocysts. Science, 282, 1145-1147.

32. Van Sloun, P. P., Jansen, J. G., Weeda, G., Mullenders, L. H., van Zeeland, A. A., Lohman, P. H., et al. (1999). The role of nucleotide excision repair in protecting embryonic stem cells from genotoxic effects of UV-induced DNA damage. Nucleic Acids Research, 27, 3276-3282.

33. Wang, Y., Medvid, R., Melton, C., Jaenisch, R., \& Blelloch, R. (2007). DGCR8 is essential for microRNA biogenesis and silencing of embryonic stem cell self-renewal. Nature Biotechnology, 39, 380-385.

34. Xu, C., Inokuma, M. S., Denham, J., Golds, K., Kundu, P., Gold, J. D., et al. (2001). Feeder-free growth of undifferentiated human embryonic stem cells. Nature Biotechnology, 19, 971-974.

35. Xu, D., Wilson, T. J., Chan, D., De Luca, E., Zhou, J., Hertzog, P. J., et al. (2002). Ets1 is required for p53 transcriptional activity in UV-induced apoptosis in embryonic stem cells. EMBO Journal, 21, 4081-4093. 